\title{
LA REFLEXIÓN COMO MEDIO DE APRENDIZAJE DE LA PRÁCTICA EDUCATIVA, TERAPEÚTICA E INVESTIGADORA
}

\author{
Adela ZAHONERO ROVIRA ${ }^{1}$
}

\begin{abstract}
RESUMEN: La Investigación Educativa, se nutre en general de los logros positivos en sus indagaciones. Al menos, es esta la información que de modo habitual vemos recogida en la literatura de una forma más extensa. Sin embargo, muchas de las cuestiones que no son interpretadas ni forman parte de los informes finales de sus conclusiones, suponen errores de los que aprender, adaptaciones a nuevas situaciones y quizá pequeños senderos de luz en un camino tan plagado de variables como es el de la Investigación Cualitativa que tiene lugar en un Centro Educativo. La reflexión sobre los aspectos que nos vemos obligados a modificar a lo largo del camino, y todas aquéllas cosas que no han salido como esperábamos pueden ser un material enormemente aprovechable para futuras experiencias, que no debemos obviar.
\end{abstract}

PALABRAS CLAVE: Investigación Acción. Contexto Educativo. Proceso de aprendizaje. Investigación Cualitativa. Adaptación. Reflexión. Atención a la Diversidad.

\section{Introducción}

El trabajo con seres humanos en contextos educativos y terapéuticos está expuesto a una enorme cantidad de variables, que, si pretendemos llevar a cabo una investigación, podrían dar al traste con la planificación mejor diseñada que hubiera cabido imaginar.

En muchos casos es la propia falta comprensión y adaptabilidad ante esa constante variabilidad en la que pueden desarrollarse las coordenadas de una investigación/ intervención pedagógica o terapeútica, la que lleva a la sensación de que la situación nos sobrepasa y al desánimo. Las investigaciones de las que hablamos, se centran en el seguimiento de herramientas diseñadas como estrategia con la finalidad de convertirse en Planes de Convivencia en Centros de Secundaria, y ponen de manifiesto que el conocimiento de esas variables que no dependen de nosotros puede ayudar a que manejemos mejor aquéllas sobre las que sí podemos influir.

Con todo, persistirán las posibilidades de que los resultados de nuestra investigación/ intervención no coincidan con lo que esperábamos o lo hagan tan sólo parcialmente.

Si es así, esta es una parte que pocos informes de investigación recogen, y aunque pueden formar parte del proceso de decisiones que nos hemos visto forzados a adoptar para acomodar la investigación a las “decisiones de reciclaje” (STUFFLEBEAM, 1987), y en todo

\footnotetext{
${ }^{1}$ Universidad de Alcalá. Departamento de Didáctica -Área de Didáctica y Organización Escolar. Guadalajara Castilla-La Mancha - España. adela.zahonero@uah.es
} 
caso ¿por qué no volver a la reflexión sobre la valoración de esas propias decisiones introducidas a lo largo del proceso para rectificarlo y/o mejorar? .

Más aún, aunque seamos personas inquietas, acostumbradas a investigar en el contexto educativo y a poner en marcha iniciativas con el objetivo de que la Convivencia mejore, precisamos de profilaxis periódicas que sometan nuestra forma de ser y actuar a cuestionamientos a fin de liberarnos de nuestros propios estereotipos. Para ello, debemos estar dispuestos a reflexionar profundamente también sobre dónde nos encontramos nosotros mismos, y cómo eso influye en lo que estamos haciendo.

En nuestra revisión sobre la literatura científica al respecto, pocas son las referencias que se detienen en la riqueza de las reflexiones en torno a los resultados no positivos de las investigaciones, las que rodean a las decisiones y rectificaciones que se hacen a o largo del desarrollo de las mismas. Obviamente la filosofía de la Investigación-Acción conlleva en sí misma la idea de la continua revisión de la espiral autoreflexiva (ABRAMS, 2005), tal y como se verá a continuación, aunque pocos informes desarrollados dentro de sus coordenadas recogen aspectos como los indicados, es en ella donde podemos ubicar esta invitación a la reflexión debate, sobre algo que corremos que puede enriquecer nuestra práctica investigadora y docente.

\section{La Investigación Acción: Reflexión sobre la propia práctica, reto y compromiso]}

Cuando Fullan (1995), define el desarrollo profesional docente utiliza el término compromiso moral, íntimamente vinculado al acuerdo establecido con la escuela para sumergirse en la práctica reflexiva, colaborativa, y en el desarrollo de una cultura de inquietudes y mejora continuas.

Con esa intención, un grupo de docentes o uno sólo (si bien siempre es mucho más efectivo trabajar de forma cooperativa a lo largo de todo el proceso), participan en el desarrollo de un programa cuyo propósito fundamental podría resumirse en la comprensión más acabada de un problema determinado a través de:

- La evaluación de la propia práctica pedagógica

- Los refuerzos y medidas pedagógicas extraordinarias de atención a la diversidad precisas dentro de los centros.

- Los apoyos terapéuticos que sean precisos a los miembros de la Comunidad Educativa. 
- El perfeccionamiento docente

- El mejoramiento de la enseñanza

- La mejora de los resultados de los aprendizajes.

- Las actividades diseñadas para procurar la mejora de la Convivencia y una consolidación de actitudes de respeto y tolerancia entre todos los miembros de la Comunidad educativa.

La investigación Acción se caracteriza fundamentalmente por orientarse hacia la mejora de la calidad educativa, y dentro de ella a sus principales agentes, los profesores como docentes- investigadores. Es esta una de sus novedades, la del nuevo tipo de investigador-a: que desde su propia realidad intenta contribuir a la resolución de problemas, cambiar y mejorar las prácticas educativas. La Investigación Acción, en la que el profesor investigador es el principal agente investigador (y por eso su experiencia de primera mano es tan importante), muestra las siguientes características:

- $\quad$ Partir de la práctica, desde la realidad situacional de los que en ella está implicados, construyendo y reconstruyendo significados.

- Tiene el fin de crear conocimiento colectivo para la acción colectiva. Si bien puede desarrollarse individualmente, su verdadero potencial se logra con el trabajo en equipo.

- Frecuentemente el grupo de personas que realizan la investigaciónacción forman parte de una comunidad o de una institución y estas personas están involucradas en la interrogación y la comprensión de su experiencia con el fin de cambiarla. Se orienta hacia la generación de procesos de reflexión crítica que implica la transformación de supuestos, marcos de referencia, puntos de vista, actividades y conductas.

- $\quad$ Se desarrolla siguiendo una espiral de ciclos de: planificación de la acción, observación sistemática, reflexión y posteriormente una planificación que facilita el paso a nuevas observaciones y reflexiones.

- $\quad$ Se puede recurrir a métodos cualitativos y cuantitativos y emplear diferentes técnicas e instrumentos de planificación, recolección de información, observación y evaluación.

- Aunque pueda ocupar distintos métodos de la investigación tradicional, su fin principal es producir conocimiento práctico y útil. 

considerada la investigación científica.

En el siguiente cuadro se muestra una propuesta de trabajo a modo de ejemplo de Aplicación de un Proyecto de Musicoterapia dentro del Aula de Convivencia que tendrá lugar el próximo curso (2009/10) en el Instituto de Enseñanza Secundaria “Lázaro Carreter” de Rivas Vaciamadrid ${ }^{2}$ :

Tabla 1: Correlación entre algunos de los Objetivos de la Musicoterapia y los del Proyecto del Aula de Convivencia ${ }^{3}$

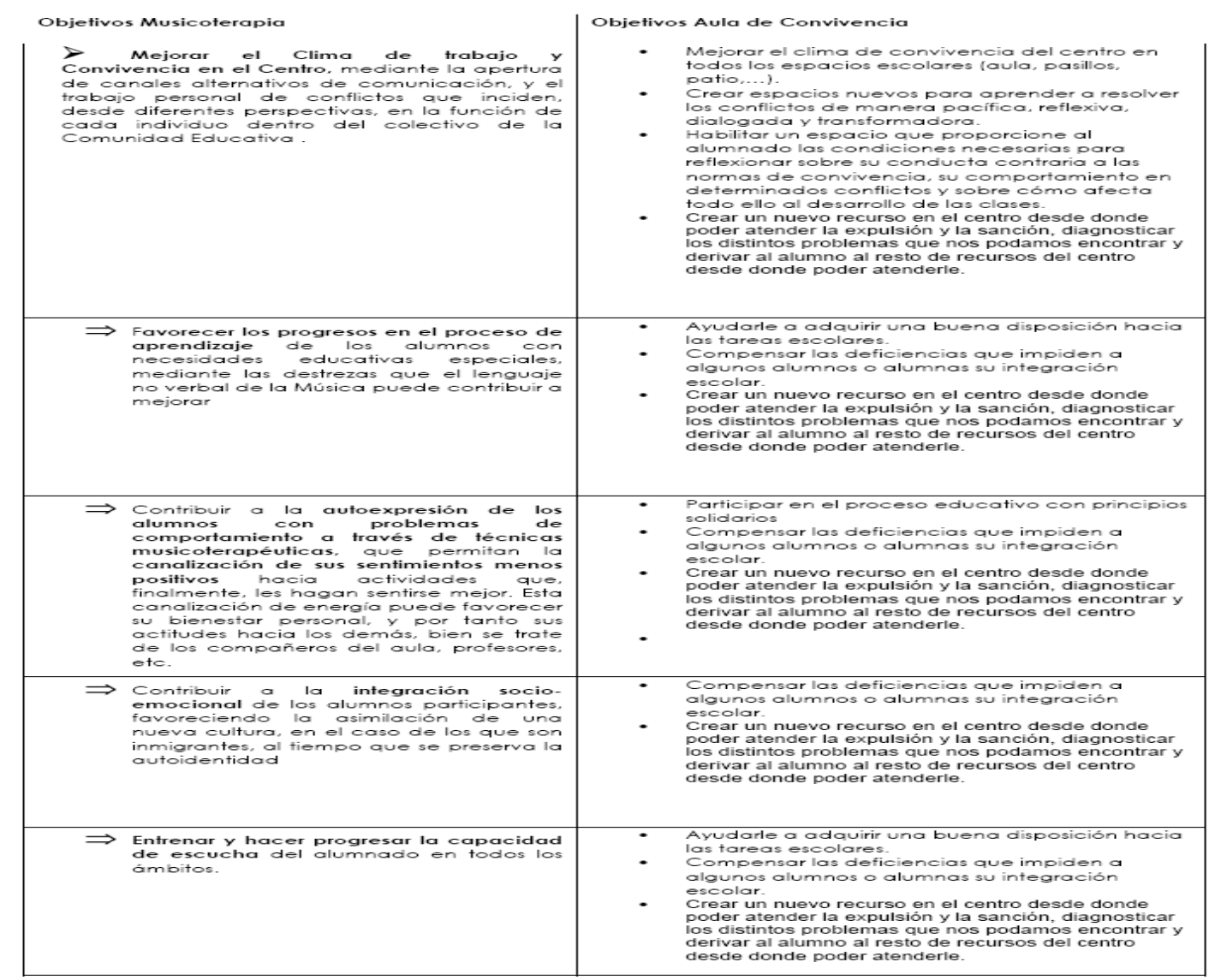

Como ya hemos referido, los procesos reflexivos son inherentes a las investigaciones, pero sin embargo sus contenidos menos positivos no suelen explicitarse de un modo generoso en los informes, y podrían recoger información importante, extrapolable a otras situaciones, y otros contextos también educativos (a otros Centros) o al mismo. Por otro lado en nuestros Institutos son relativamente frecuentes investigaciones centradas en Estudios de Casos o

\footnotetext{
${ }^{2}$ Información procedente del mencionado Proyecto, y cedida por D.Juan Carlos Hervás, Jefe de Estudios del Instituto y, autor del Proyecto del Aula de Convivencia, y de los objetivos que aquí se recogen en el margen derecho. Adela Zahonero planteó en la tabla los que, dentro de la aplicación de Musicoterapia pueden relacionarse con cada apartado del Aula de Convivencia.
} 
Caso único, investigaciones marcadamente etnográficas resultado de las situaciones tan paradójicas y variables que podemos encontrar en un centro educativo. Si, lógicamente hemos de conseguir que nuestra investigación esté, según hemos apuntado más arriba sistematizada y responda a unos determinados criterios de validación para que pueda ser considerada científica, estamos llegando a un punto álgido de la reflexión cuando se trata de determinados contextos. Realmente, las conclusiones de la investigación, serán, más bien verdades, en plural que una única verdad. En un artículo muy influyente, David Bridges (1999), afirma, que la teoría pragmática de la verdad es la que uno se encuentra en la realidad de la investigación acción en Aula. Sin embargo, esto podría incluso no tener que ver con la investigación acción. Heikkenin, Kakkori, Huttenen (2001, p.22) aporta directamente un análisis centrado en la investigación acción, que muestra que la teoría práctica de la verdad que realmente funciona tiene un uso limitado. Su razonamiento, que compartimos, es que la mayor parte de “aspectos de la verdad tienen puntos válidos y otros con restricciones”, sobre todo dentro del contexto escolar.

Los ambientes escolares dan lugar a realidades multiparadigmáticas, y consecuentemente a situaciones muy diversas e incluso paradógicas para los investigaciones en la acción, lo que podría ser analizado como situación muy productiva, ahora bien, desde un punto de vista científico más "purista” es obvio que lleva a la conclusión de que la investigación Acción supone un estadio poco desarrollado de la Ciencia, poco riguroso. Yendo un poco más allá, sería posible relacionar las pequeñas historias buscando coherencia en su diseño y por tanto, entre las distintas verdades que arrojan.

\section{Metodología: Algunos ejemplos en torno a nuestra propuesta}

La selección de los métodos de investigación depende de los propósitos y de las preguntas de investigación. Los principales recursos técnicos son:

- El diario personal o registros

- La entrevista

- El grupo focal (grupo de docentes y guía de preguntas)

- La encuesta

- La observación

- El análisis de documentos

- La grabación (video, audio, fotografía) 
- La muestra, escasamente utilizada en investigación cualitativa. En investigaciónacción debe permitir profundizar en la comprensión sobre una situación con un grupo determinado con el fin de generar un cambio en dicho grupo.

Posibles errores en la metodología previos a la puesta en marcha de la Investigación

Entre los errores más frecuentemente cometidos, suelen situarse en muchos casos el modo en que la Investigación se presenta a determinados sectores de la Comunidad Educativa, y la posición que, sintiéndonos investigadores, adoptamos ante otras personas que juzgamos menos “doctas” en materia educativa: El tipo de lenguaje con el que informemos de nuestros propósitos a todas las personas a quienes queremos involucrar en un proyecto ilusionante, será fundamental, y me refiero especialmente a los padres de nuestros alumnos y a los propios alumnos. Pero además, cada uno, en esta cadena, debe verse investida de la dignidad de la responsabilidad y la confianza que se le otorga a su participación, y sentirse respetado e importante por ello en el compromiso que adquiere, de donde se deriva además cuidar con un tacto exquisito todo lo concerniente a las relaciones humanas que se establecen en los centros como premisa a cualquier propuesta, y refuerzo constante a lo largo de toda la reflexión que retroalimentará y guiará la evolución de la investigación en la que se fundamenta.

Los criterios de selección de las personas participantes en el Proyecto o en la Investigación, también pueden generar problemas por conflictos de intereses, así como si no hay una absoluta transparencia entre los papeles de quienes coordinen la investigación y su actuación de cara a los demás. Si bien, por criterios de selección también nos referimos, naturalmente, a las decisiones más o menos colegiadas, que hacen que determinados alumnos estén agrupados de determinada forma, participen en programas concretos, o reciban apoyos específicos. Por lo general suelen ser estos dos últimos los capítulos más delicados y sujetos a continua revisión.

Muchos Proyectos destinados a la mejora de la Convivencia gravitan en torno a la oferta de programas específicos para alumnos con problemas emocionales que repercuten en su adaptación socioeducativa y en su aprendizaje, y así nos encontramos desde actividades diseñadas para las llamadas “Aulas y talleres de Convivencia”, a la aplicación de Musicoterapia u otras terapias artísticas y creativas, y otros tipos de propuestas que son constitutivas de distintas alternativas de investigación. En casi todos los casos, los profesionales responsables de llevar a cabo el proyecto suelen formar parte de la Comunidad 
Educativa, y diseñar cuidadosamente la actividad, siendo ellos mismos quienes están al frente del equipo de investigación Acción, si bien deben afrontar otro de los errores que se producen con frecuencia en este tipo de contextos y con esta tipología de proyectos, y se trata del escaso margen de participación que normalmente por razones organizativas, les es concedido para seleccionar a los alumnos, decidir como agruparlos, y la poca flexibilidad que el horario escolar y sus sesiones horarias otorgan a actividades que no se adapten a los 55 minutos de los periodos lectivos habituales. Este es también otro punto que incide en la reflexión porque afecta al desarrollo de cualquier idea innovadora dentro de la organización escolar, y puede tener su reflejo en la investigación.

Recursos técnicos: los aspectos especialmente vulnerables

En las Investigaciones dirigidas a promover una Convivencia más armónica cuidar todos los detalles inherentes a tratar con la máxima exquisitez posible las relaciones humanas, y en consecuencia, es fundamental subrayar que debiera ser la máxima aspiración. Por ello, los recursos técnicos que se utilizan en las indagaciones, como por ejemplo las grabaciones audiovisuales, deben manejarse del modo menos invasivo posible para las personas a las que se vaya a grabar, explicando claramente la finalidad de la grabación dentro de la investigación y garantizando absolutamente la confidencialidad de las imágenes (ZAHONERO ROVIRA, 2007).

Los recursos e instrumentos responden a un diseño general de Investigación fundamentalmente cualitativo, (aunque puedan tener cabida datos cuantitativos), que suponen una implicación personal de todos los participantes en la investigación mucho mayor, y al mismo tiempo, la necesidad de que esas implicaciones interfieran en los resultados de la investigación lo menos posible.

Se trata de una invitación para actuar en el silencio y es de allí, escuchar nuestras propias voces y los ecos del pasado, de la historia, del presente, de la eventualidad.

Invitación a sentir y a presentir la ciencia en su rigor imponente que la caracteriza y con ella, descifrar los misterios que habitan más allá de los hechos que observamos. Un docente que logre estos ambientes educativos, será capaz de conjugar, desarrollar y gestionar sus currículos desde la relación con la investigación. Una relación que demanda acción donde el actor, docente, intervenga y provoque la participación real de los invitados. Allí donde conocimiento, realidad y sujetos -que como signos, que forman parte de un macrosignoactúen, establezcan relaciones y dinamicen el aprendizaje. 
Este relacionarse concientemente, como interacción, provoca transformaciones en la manera de asumir la realidad, de comprendernos como sujetos y de comprender la ciencia para esclarecer ideas, realidades, anhelos.

\section{Complejidad del Contexto de Investigación-Acción}

El contexto educativo cambia constantemente. Definir una investigación en su seno es algo parecido al dique de Osteschelde, en la región holandesa de Zeelandia, una sapientísima mezcla de contención y flexibilidad, frente y junto al mar, un alarde de adaptación de un plan cuidadosamente diseñado, que, sin embargo fruto de una continua reflexión/ aprendizaje termina siendo algo muy distinto a lo que en apariencia su primera imagen de fortaleza rígida nos ofrecía.

Más allá del conocimiento de los intereses técnico, práctico o emancipatorio que propone Habermas y que Grundy traduce como función del currículo, los actores que participan en la escena educativa tendrán que apropiarse y asumir el currículo o los diseños innovadores como su propia realidad. Ello implica no solo conocimiento, sino también reflexión y comprensión de la realidad, de las cosas, de los seres humanos, de lo dicho y silente; sabiduría para interactuar con el otro, consigo mismo, con la ciencia; y visión de mundo presta a modificarse y a transformarse a partir de estas relaciones.

En términos generales, un diseño de Investigación podrís representarse del siguiente modo en Investigación -Acción:

\section{Diseño de la Investigación -Acción}

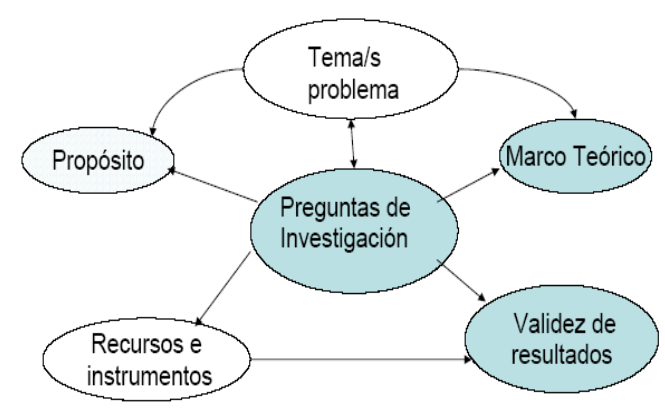

Figura 1- Disenõ de la Investigación -Acción.

Fuente: Propia. 
Llevar a cabo la investigación en un contexto de relaciones humanas requiere una planificación rigurosa y una estrecha colaboración con las personas con las que se debe tratar. La mayor parte de los Proyectos de mejora de Convivencia comprometen a alumnos con diversas problemáticas en su equipaje vital, traducidos en conductas disruptivas de distinta índole, pero además involucra a sus padres como elemento esencial en tanto al papel que juegan dentro de su entorno familiar, como por lo que como miembros de la Comunidad Educativa del Centro se refiere. Es más que probable, que también haya profesores enrolados en proyectos de Convivencia, como resultado de las tensiones que se generan en las Instituciones educativas ante la reiteración de ambientes conflictivos y la necesidad de buscarles una salida. En algunos casos, estos programas cuentan además con la participación del personal no docente. De un modo u otro, en un lugar en el que las interactuaciones son tantas, las interferencias acompañan exponencialmente.

Los investigadores pueden encontrar dificultades para hacer frente a aspectos inesperados de una situación real, independientemente de lo bien planificada que haya sido una investigación.Algunos de estos pueden ser:

1. La selección de las personas que forman parte del Proyecto sobre el que se investiga.

2. La regularidad de asistencia de esas personas a la actividad. Muchos de los alumnos que suelen seleccionarse tiene un largo historial de absentismo, otros problemas de enfermedad de larga duración que distorsionan su propia participación y la calidad de sus interacciones con el grupo.

3. En ciertos casos se incorporan a los programas personas nuevas que también distorsionan la marcha conjunta de los agrupamientos.

4. Buen número de los alumnos participantes son hijos inmigrantes, y en mitad de la investigación su familia decide volver a su país o cambiar de lugar de residencia, lo que provoca que sean una baja inmediata en los datos de la investigación.

5. Los niveles de compromiso participación de los padres en el seguimiento y participación en los proyectos resultan, por lo general, más bajos de lo esperado, si bien tampoco es de extrañar, ya que muchos de los 
alumnos participantes proceden de familias desestructuradas con poco o muy escaso contacto con los Centros.

6. Suele ser habitual que las madres estén más abiertas a la participación y cooperación, con el Centro y con el /los investigadores, mientras que la participación de los es más aislada.

7\%. En algunos Proyectos de investigación, se crean problemas añadidos tales como:

- Necesidad de espacios que colisionan con otras actividades “normales” de la vida académica del centro.

- Ruidos, alteraciones del Clima óptimo de aprendizaje para los demás miembros de la Comunidad educativa. Si se desarrolla un proyecto de Aplicación musicoterapéutica, por ejmplo, en determinados momentos, las expresiones sonoras de los participantes pueden llevar a una intensidad que perturbe a los demás, si el lugar en el que se desarrollan las sesiones no está convenientemente asilado desde el punto de vista acústico.

- $\quad$ Posibles tensiones a la hora de tomar decisiones acerca de la conveniencia de la participación de un alumno en alguna actividad cuando coincida dentro del horario lectivo con profesores que consideran que su asignatura debiera ser más importante.

\section{A modo de Conclusión}

En Stenhouse (1996) asistimos continuamente a una unión entre teoría y práctica en el terreno docente. Nosotros también creemos que ese es el marco adecuado para asentar los estudios sobre didáctica y educación. La experiencia directa aporta unos tintes indelebles, enormemente ricos, y es por ello, que aún siguiendo a Stenhouse daríamos un paso más allá para situar la autocrítica surgida de la investigación en el plano del aprendizaje, tanto como para que no nos duela seguir volviendo sobre él, reflexionando acerca de cuanto positivo y negativo nos hemos encontrado en el camino y compartirlo todo, también lo no positivo, al igual que otras cosas que quizá evitan alguna vez el tener que "escarmentar en cabeza propia”. 


\section{REFLECTION AS A RESOURCE OF LEARNING IN THE EDUCATIONAL, THERAPEUTICAL AND RESEARCHAL PRACTICE}

ABSTRACT: Educational research is based on general results from succesful achievement. on his inquiries. In an extended way, this is the information we see collected in literature.However, most issues that might not appear or even be reflected on concluding reports can be taken as errors to improve and adaptations to new situations. In adition to this, they would be considered as a throwing light" over such a plague variable path as the Cualitative research is when taking place in an educational context. Reflection upon the aspects forced to be changed along the way, plus all those things that have not come as we expected, (those events not resulting as they were expected) can be a highgly exploitable material for future experiences, that we might not ignore.

KEYWORDS: Action Research. Educational Context. Learnin. Process. Cualitative research. Adaptation. Reflection. Attending diversity.

\section{REFERENCIAS}

ABRAMS, B. Evaluating Qualitiative Music Therapy Research. In: WHEELER, B. L. (Ed.). Music Therapy Research. Gilsum: Barcelona Publishers, 2005. p.246-258.

BRIDGES, D. Educational research: pursuit of truth or flight into fancy? British Educational Research Journal, Oxfordshire, v.25, n.5, p.597-616, dec. 1999.

FULLAN, M. The limits and the potential of professional development. In: GUSKEY T. R.; HUBERMAN, M. (Ed.). Professional development in education: new Paradigms and Practices. New York: Teachers College Press, 1995. p.253-267.

GRUNDY, S. Producto o praxis del currículo. Madrid: Morata, 1998.

HEIKKENIN, H.; KAKKORI, L.; HUTTUNEN, R. This is my truth, tell me yours: some aspects of action research quality in the light of truth theories. Educational Action Research, Wallingford, v.9, n.1, p.9-24, 2001.

STENHOUSE, L. La investigación como base de la enseñanza. Madrid: Morata, 1996.

STUFFLEBEAM, D. L. SHINKFIELD, A. J. Evaluación Sistemática, Guía Teórica y

Práctica. Barcelona: Paidos, 1987.

ZAHONERO ROVIRA, A. La atención a la Diversidad desde la Musicoterapia. Revista Iberoamericana de Educación, Madrid, v.2. n.2, p.1-18, 2007. Disponible en:

$<$ www.fclar.unesp.br/seer/index.php?journal=iberoamericana\&page=index>. Acceso en: 19 nov. 2008. 


\section{BIBLIOGRAFÍA CONSULTADA}

BOGGINO, N.; KRISTIN, R. Investigación-acción-reflexión crítica sobre la práctica educativa: orientaciones prácticas y experiencias. Madrid: Trillas Eduforma, 2007.

BRAY, J. N. et al. Collaborative inquiry in practice: action, reflection, and making meaning. Thousand Oaks: Sage Publications, 2000.

COTTON, T.; GRIFFITHS, M. Action research, stories and practical philosophy.

Educational Action Research, Wallingford, v.15, n.4 p.545-560, dec. 2007.

ELLIOTT, J. Action research for educational change. Buckingham: Open University Press, 1991.

FULLAN, M.; HARGREAVES, A. Teacher development and educational change.

London: Falmer, 1992.

HERRÁN GASCÓN, A.; GONZÁLEZ SÁNCHEZ, I. El ego docente, punto ciego de la enseñanza: el desarrollo y la formación del profesorado. Madrid: Universitas, 2001.

McKERNAN, J. Investigación-acción y currículo. Madrid: Morata, 2001.

KEMMIS, S. Exploring the relevance of critical theory for action research: emancipatory action research in the footsteps of Jurgen Habermas. In: REASON, P.; BRADBURY, H. (Ed). Handbook of action research: participative inquiry and practice. London: Sage, 2001. p.91102.

PARK, P. Knowledge and participatory research. In: REASON, P.; BRADBURY, H. (Ed.). Handbook of action research: participative inquiry and practice. London: Sage Publications, 2001. p.81-90.

ROGERS, C. R. On becoming a person: a therapist's view of psychotherapy. Boston: Houghton Mifflin, 1961.

ROWAN, J. The Humanistic approach to action research. In: BRADBURY, P. R. H. (Ed.). Handbook of action research: participative inquiry and practice. Thousand: Sage Publications, 2001. p.114-123.

SMITH, L. Ethical principles in practice: evidence from participatory action research.

Kairaranga, [S.l.], v.9, Special Edition, p.16-21, 2008. 